\title{
Obtención de un filtrante de anís de monte (Tagetes filifolia Lag.) edulcorado con hojas de estevia (Stevia rebaudiana Bertoni)
}

\section{Obtaining a filter irish lace marigold (Tagetes filifolia Lag.) sweetened with stevia leaves (Stevia rebaudiana Bertoni)}

\author{
Carlos Millones ${ }^{1}$, Gerardo Mori ${ }^{2}$, Joel Bacalla ${ }^{2}$, Ernestina Vásquez ${ }^{1}$, Rocío Tafur ${ }^{1}$ \\ ${ }^{1}$ Laboratorio de Biología, Universidad Nacional Toribio Rodríguez de Mendoza de Amazonas. Ciudad Universitaria. El \\ Franco, Higos Urco, Chachapoyas-Amazonas. \\ ${ }^{2}$ Escuela de Ingeniería Agroindustrial, Universidad Nacional Toribio Rodríguez de Mendoza de Amazonas.
}

Recibido 10 noviembre 2013. Aceptado 10 marzo 2014.

\begin{abstract}
Resumen
El presente trabajo de investigación se realizó para determinar el segmento de la planta de anís de monte y formulación apropiada de anís de monte y hojas de estevia en la elaboración de un filtrante edulcorado; para lo cual se emplearon plantas de anís de monte y hojas de estevia, las cuales fueron lavadas, desinfectadas, secadas a $65^{\circ} \mathrm{C}$, molidas, tamizadas y envasadas; posteriormente se realizaron las formulaciones del filtrante con anís de monte y hojas de estevia (90:10; 85:15; 80:20 y 75:25), empleando un gramo por muestra envasadas en papel termosellable; los resultados del color, tiempo de infusión y pH; asimismo, las evaluaciones organolépticas de olor, sabor, dulzor y aspecto general se procesaron empleando el paquete estadístico SAS (Statistical Analysis System) for Window V8. Los resultados mostraron que empleando 80 a $85 \%$ hojas + flores de anís de monte y 15 a $20 \%$ de hojas de estevia se obtiene un filtrante edulcorado con adecuadas características organolépticas.
\end{abstract}

Palabras clave: Filtrante, estevia, edulcorante, esteviósido, aceite esencial.

\begin{abstract}
The present investigation was conducted to determine the segment of the irish lace marigold and appropriate formulation of irish lace marigold and leaves of stevia in the development of a filter sweetened, for which plants were used irish lace marigold and leaves stevia, which were washed, disinfected, dried at $65{ }^{\circ} \mathrm{C}$, ground, sieved and packed; later formulations were made with irish lace marigold and stevia leaves (90:10, 85:15, 80:20 and 75:25), using a gram per sample packed in sealable paper, the results of the color, infusion time and $\mathrm{pH}$, also the organoleptic evaluations smell, taste, sweetness and overall appearance were processed using the SAS statistical package (Statistical Analysis System ) for Window V8. The results showed that using 80 to 85 $\%$ leaves + flowers irish lace marigold and 15 to $20 \%$ stevia leaf with a sweetened organoleptically suitable filter is obtained.
\end{abstract}

Keywords: Filter, stevia, sweetener, estevioside, essential oil.

\section{Introducción}

La región Amazonas se caracteriza por poseer una serie de microclimas que hace posible el desarrollo de una diversidad de especies vegetales, entre las que destaca el anís de monte que desarrolla naturalmente en las zonas altoandinas de la región Amazonas. Asimismo, la estevia que es cultivada en las zonas subtropicales de la provincia de Rodríguez de Mendoza, región Amazonas. Ambas especies poseen un potencial para su agroindustrialización. Tagetes filifolia Lag. (Tribu Tageteae, Familia Asteraceae), conocida como "anís mexicano", "anisillo" o "anís de monte" es una planta anual de porte bajo que alcanza 5 a $50 \mathrm{~cm}$ de altura según la densidad de población y las condiciones del suelo; es herbácea y aromática, de condición silvestre ruderal o viaria, con un

\footnotetext{
* Autor para correspondencia

E-mail: carlos.millones@untrm.edu.pe (C. Millones)
} 
rendimiento de aceite cercano a $1 \%$ (Campos et al., 2010), cuyos compuestos principales del aceite esencial son anetol o alilanisol (Serrato-Cruz et al., 2008), el $\alpha$ terthienyl compuesto alelopático para muchas especies de fitonemátodos (Cerruti et al., 2010). Asimismo, el aceite esencial de anís de sierra presenta actividad antibacteriana del tipo bactericida contra Escherichia coli y Salmonella typhimurium al emplear una concentración del 2,5 y 5\% (Vásquez et al., 2011).

La Stevia rebaudiana Bertoni, es un antiguo arbusto perenne de América del Sur, produce glucósidos de diterpeno que son los edulcorantes bajos en calorías, alrededor de 300 veces más dulce que la sacarosa. Extractos de Stevia, además de tener propiedades terapéuticas, contienen un alto nivel de compuestos edulcorantes, conocidos como glucósidos de esteviol, que se cree que poseen actividad antioxidante, antimicrobiana y antifúngica. El esteviósido y el rebaudiósido A son los principales compuestos edulcorantes de interés. Ellos son termoestables, incluso a temperaturas de hasta $200{ }^{\circ} \mathrm{C}$, que los hace adecuados para su uso en los alimentos cocinados. S. rebaudiana tiene un gran potencial como un nuevo cultivo agrícola ya que la demanda de los consumidores de alimentos a base de hierbas está aumentando y análisis proximal ha demostrado que la Stevia también contiene ácido fólico, vitamina $\mathrm{C}$ y de todos los aminoácidos indispensables con la excepción de triptófano (LemusMoncada et al., 2012). La infusión de hojas de estevia 1,5 a 2,5\% (w/v) posee una actividad antibacteriana en el crecimiento de Listeria innocua (BeldaGalbis et al., 2014); asimismo, el empleo del $2,5 \%$ (w/v) de $S$. rebaudiana inactiva más de 5 ciclos de registro de Listeria monocytogenes y la máxima inactivación de la polifenoloxidasa con la mayor retención de los componentes bioactivos en extractos de frutas procesadas (Barba $e t$ al., 2014); entretanto, las mezclas combinadas de $S$. rebaudiana y jugos de frutas pueden ser una herramienta útil para proporcionar nuevos productos alimenticios con mayores propiedades nutritivas (Carbonell-Capella et al., 2013).

En la actualidad, existe una tendencia al uso de infusión de plantas aromáticas, debido a que se considera una bebida buena para la salud; es así, que se han desarrollado filtrantes a partir de hierba luisa (Vásquez, 1987); hojas de sauco (Ortíz et al., 2006); rizoma de jengibre (Acuña y Torres, 2010); maíz morado (Nolazco, 2008); mezcla de manzanilla, hierba luisa y uña de gato (Follegatti, 2002).

Por lo antes considerado se planteó la presente investigación con la finalidad de aprovechar agroindustrialmente plantas de anís de monte y hojas de estevia en la elaboración de un filtrante edulcorado; en tal sentido se plantearon como objetivo determinar la parte de planta de anís de monte, y formulación apropiada de anís de monte y hojas de estevia en la elaboración de un filtrante edulcorado que posea un adecuado grado de aceptabilidad.

\section{Material y métodos}

En la presente investigación se empleó como materia prima plantas de anís de monte (Tagetes filifolia Lag.) en un 50\% de floración provenientes de los huertos de la ciudad de Chachapoyas, y hojas de estevia (Stevia rabaudiana Bertoni) proveniente de la provincia de Rodríguez de Mendoza, región Amazonas; las cuales fueron llevados a los laboratorios de la Universidad Nacional Toribio Rodríguez de Mendoza de Amazonas para los ensayos respectivos para la elaboración de un filtrante de anís de monte, edulcorado con hojas de estevia.

Para la obtención de hojas deshidratadas de estevia, se recolectaron hojas frescas de plantas de estevia de aproximadamente 60 $\mathrm{cm}$ de longitud, las cuales se lavaron y se remojaron en una solución de lejía Clorox al $5 \%$ por alrededor de 30 minutos, posteriormente se enjuagaron con agua 
corriente y se colocaron en una estufa Marca Nahita a temperatura de $65^{\circ} \mathrm{C}$ hasta que las hojas alcanzaron una humedad de 6\%; posteriormente se molieron en un molino con tornillo sin fin marca Corona y se colocaron en una bolsa de plástico de alta densidad para su posterior uso en la elaboración del filtrante.

Para la obtención de plantas deshidratadas de anís de monte, se recolectaron plantas en un $50 \%$ de floración las cuales se lavaron y remojaron en una solución de lejía Clorox al 5\% por alrededor de 30 minutos, se enjuagaron con agua corriente, se orearon durante 3 horas y se colocaron en una estufa Marca Nahita a temperatura de $65^{\circ} \mathrm{C}$ hasta que las plantas alcanzaron una humedad de 6\%; posteriormente se separaron las plantas en a1: Hojas + flores; a2: tallos y a3: planta completa; luego se molieron en un molino con tornillo sin fin marca Corona, se tamizaron para eliminar las partículas grandes empleando un tamiz 90; posteriormente se colocaron en una bolsa de plástico de alta densidad para su posterior uso en la elaboración del filtrante.

Una vez obtenidas las hojas de estevia y plantas de anís de monte molidas se realizaron las combinaciones siguiendo los tratamientos mostrados en la Tabla 1; pesando las muestras en una balanza de precisión ES-300, siendo el peso total del filtrante de 1 gramo y empleándose papel termosellable color blanco-Ref-746, con dimensiones de $6 \mathrm{~cm}$ de alto por $5,5 \mathrm{~cm}$ de ancho. Una vez colocadas las muestras en las bolsitas, se procedió al sellado por las partes laterales de estas, y la parte superior, después de haber colocado el hilo sostén, el cual fue previamente desinfectado en lejía Clorox al 5\%; una vez embolsadas las muestras, se empacaron en bolsas de plástico de alta densidad para posteriormente realizar las evaluaciones fisicoquímicas y organolépticas del filtrante obtenido.

Para la evaluación de los datos de color se empleó un espectrofotómetro JENWAY640 UV/VIS empleando absorbancia a 380,430 y $480 \mathrm{~nm}$.
En la determinación del tiempo de infusión se empleó $1 \mathrm{~g}$ de la muestra colocada en un vaso de precipitación de $150 \mathrm{~mL}$ cubierto con un placa Petri, al cual se agregó 100 $\mathrm{mL}$ de agua hirviente, iniciando a contabilizar el tiempo desde el inicio de la infusión hasta la extracción máxima de compuestos, dichas determinaciones se realizaron a los tratamientos empleados y a filtrantes comerciales manzanilla y anís Herbi, manzanilla y anís McCollins.

\section{Tabla 1}

Combinaciones de segmentos de la planta de anís de monte ( $T$. filifolia Lag.) y hojas de estevia ( $S$. rebaudiana B.) en la elaboración de un filtrante

\begin{tabular}{cccc}
\hline & Segmento de la & \multicolumn{2}{c}{ Formulación } \\
\cline { 3 - 4 } Trata- & planta de anís de & Anís & Hoja \\
miento & monte empleada & de \\
& en el filtrante & $\begin{array}{c}\text { de } \\
\text { monte } \\
(\mathrm{g})\end{array}$ & $\begin{array}{c}\text { estevia } \\
(\mathrm{g})\end{array}$ \\
\hline T1 & Hoja + flor & 0,90 & 0,10 \\
T2 & Hoja + flor & 0,85 & 0,15 \\
T3 & Hoja + flor & 0,80 & 0,20 \\
T4 & Hoja + flor & 0,75 & 0,25 \\
T5 & Tallos & 0,90 & 0,10 \\
T6 & Tallos & 0,85 & 0,15 \\
T7 & Tallos & 0,80 & 0,20 \\
T8 & Tallos & 0,75 & 0,25 \\
T9 & Planta completa & 0,90 & 0,10 \\
T10 & Planta completa & 0,85 & 0,15 \\
T11 & Planta completa & 0,80 & 0,20 \\
T12 & Planta completa & 0,75 & 0,25 \\
\hline
\end{tabular}

El pH de las muestras de filtrantes de anís de monte edulcorado con hojas de estevia se midió con un potenciómetro Hanna Instruments HI8424. Para la evaluación de los datos registrados se empleó un experimento bifactorial $3 \mathrm{~A} \times 4 \mathrm{~B}$ bajo un Diseño Completamente al Azar, siendo el Factor A: segmentos de la planta de anís de monte (a1: hojas + flores; a2: tallos; a3: planta completa) y Factor B: formulación anís de monte y hojas de estevia (b1: 90:10; b2: 85:15; b3: 80:20 y b4: 75:15), evaluando mediante el análisis de varianza y para las comparaciones de medias de los tratamientos se empleó la prueba Tukey al $95 \%$ de confianza. Para la evaluación sensorial se empleó un DBCA con 12 panelistas semientrenados, quienes evalua- 
ron los atributos de olor, sabor, dulzor y aspecto general de las infusiones de los filtrantes elaborados; realizando el análisis de varianza y la prueba Tukey al $95 \%$ para la comparación de las medias. Para el procesamiento de los datos se empleó el paquete estadístico SAS (Statistical Analysis System) for Window V8.

Al mejor tratamiento de filtrante obtenido se realizó la determinación de ceniza (Método AOAC 920.100A, 2005), fibra cruda (AOAC 930.10, 2012), Humedad (NTP 209.253, 1986) y aceite esencial (método de arrastre por vapor).

\section{Resultados y discusión}

En la Tabla 2 se muestra la evaluación del color, tiempo de infusión y $\mathrm{pH}$ de la infusión del filtrante obtenido a partir de anís de monte y hojas de estevia. Con respecto al color de la infusión a una longitud de onda de 380 y $430 \mathrm{~nm}$ se registró la mayor absorbancia en los tratamientos T1, T2, T3 y T4 donde se empleó hojas + flores, cuyo color complementario de la absorbancia es de color verde-amarillo; la disminución de la intensidad del color verde-amarillo se ve reflejado en una disminución en los valores de la absorbancia registrado en los tratamientos $\mathrm{T} 9, \mathrm{~T} 10, \mathrm{~T} 11$ y $\mathrm{T} 12$, influenciado por el uso en la elaboración del filtrante con planta completa de anís de monte. El empleo de tallo de plantas de anís de monte en los tratamientos T5, T6, T7 y T8 para la elaboración del filtrante se caracterizó por registrar menores valores de absorbancia, cuyo color complementario de la absorbancia es amarillo; asimismo, al comparar el color en la tabla Munsell Soil Color Charts (2010), registró un color amarillo pálido (Tabla 3), por lo tanto, el empleo de tallos de anís de monte no influencia marcadamente en el color del filtrante. Olsen (1986) manifiesta que el color que presenta un material es el resultado de la combinación de la absorción (o reflexión) de radiación en cada una de las bandas de color del espectro visible, así, cualquier material tiene un espectro característico que nos indica la radiación que absorbe o refleja a una determinada longitud de onda de la radiación incidente, cuando la longitud de onda está comprendida entre $400 \mathrm{~nm}$ y 750 nm (zona del espectro de radiación visible), es así, que el color complementario en la absorbancia de las infusiones de los filtrantes elaborados fue de color amarillo-verde $(380$ y $430 \mathrm{~nm})$ y amarillo (480 nm), respectivamente.

\section{Tabla 2}

Evaluación del color, tiempo de infusión y pH del filtrante elaborado a partir de anís de monte (Tagetes filifolia Lag.) edulcorado con hojas de estevia (Stevia rebaudiana Bertoni).

\begin{tabular}{|c|c|c|c|c|c|c|c|c|c|c|}
\hline \multirow[t]{2}{*}{$\begin{array}{l}\text { Trata- } \\
\text { miento }\end{array}$} & \multirow{2}{*}{$\begin{array}{l}\text { Segmento } \\
\text { Planta } \\
\mathrm{AM}^{2}\end{array}$} & \multicolumn{2}{|c|}{$\begin{array}{l}\text { Formula- } \\
\text { ción }(\%)\end{array}$} & \multicolumn{4}{|c|}{ Color (absorbancia) ${ }^{1}$} & \multirow{2}{*}{$\begin{array}{l}\text { Tiempo Inicial } \\
\text { Infusión } \\
(\mathrm{s})^{1}\end{array}$} & \multirow{2}{*}{$\begin{array}{l}\text { Tiempo Final } \\
\text { Infusión } \\
(\mathrm{s})^{1}\end{array}$} & \multirow[t]{2}{*}{$\mathrm{pH}^{1}$} \\
\hline & & $\mathrm{AM}^{3}$ & $\mathrm{HE}^{3}$ & $380 \mathrm{~nm}$ & $430 \mathrm{~nm}$ & & $480 \mathrm{~nm}$ & & & \\
\hline T1 & $\mathrm{H}+\mathrm{F}$ & 90 & 10 & $2,29 \pm 0,08 \mathrm{a}$ & $0,71 \pm 0,07$ & & $0,25 \pm 0 \quad a b$ & $19,7 \pm 6,7 \mathrm{ab}$ & $266,3 \pm 57 \mathrm{ab}$ & $6,48 \pm 0,18 d$ \\
\hline $\mathrm{T} 2$ & $\mathrm{H}+\mathrm{F}$ & 85 & 15 & $2,33 \pm 0,14 \mathrm{a}$ & $0,67 \pm 0,04$ & & $0,25 \pm 0,01 \quad a b c$ & $22,0 \pm 4,6 \mathrm{ab}$ & $242,3 \pm 40 \mathrm{~b}$ & $6,59 \pm 0,10 \mathrm{~cd}$ \\
\hline $\mathrm{T} 3$ & $\mathrm{H}+\mathrm{F}$ & 80 & 20 & $2,22 \pm 0,03 \mathrm{a}$ & $0,69 \pm 0,06$ & & $0,28 \pm 0,07$ a & $24,3 \pm 1,5$ a & $263,0 \pm 27 \mathrm{ab}$ & $6,64 \pm 0,10 c$ \\
\hline $\mathrm{T} 4$ & $\mathrm{H}+\mathrm{F}$ & 75 & 25 & $2,23 \pm 0,03 \mathrm{a}$ & $0,69 \pm 0,08$ & $\mathrm{a}$ & $0,28 \pm 0,06$ a & $20,0 \pm 1,0 \mathrm{ab}$ & $277,0 \pm 16 \mathrm{ab}$ & $6,70 \pm 0,08 \mathrm{c}$ \\
\hline T5 & Tallos & 90 & 10 & $0,99 \pm 0,06 \mathrm{~d}$ & $0,28 \pm 0,08$ & a & $0,19 \pm 0,01 \quad \mathrm{c}$ & $17,0 \pm 2,7 \quad b$ & $273,3 \pm 44 a b$ & $6,84 \pm 0,02 b$ \\
\hline T6 & Tallos & 85 & 15 & $1,11 \pm 0,17 \mathrm{~d}$ & $0,36 \pm 0,08$ & a & $0,21 \pm 0,04 \quad b c$ & $22,0 \pm 7,6 \mathrm{ab}$ & $344,0 \pm 74$ a & $6,90 \pm 0,02 \mathrm{ab}$ \\
\hline $\mathrm{T} 7$ & Tallos & 80 & 20 & $1,10 \pm 0,10 \mathrm{~d}$ & $0,37 \pm 0,03$ & & $0,21 \pm 0,02$ bc & $20,0 \pm 4,6 \mathrm{ab}$ & $302,7 \pm 93 \mathrm{ab}$ & $6,94 \pm 0,06 \mathrm{a}$ \\
\hline $\mathrm{T} 8$ & Tallos & 75 & 25 & $1,13 \pm 0,03 \mathrm{~d}$ & $0,38 \pm 0,02$ & & $0,22 \pm 0,03 \mathrm{abc}$ & $15,7 \pm 3,1 b$ & $344,0 \pm 54$ a & $6,98 \pm 0,02 \mathrm{a}$ \\
\hline $\mathrm{T} 9$ & PC & 90 & 10 & $1,57 \pm 0,03$ bc & $0,44 \pm 0,02$ & & $0,22 \pm 0,03 \mathrm{abc}$ & $20,3 \pm 4,5 \mathrm{ab}$ & $288,3 \pm 23 \mathrm{ab}$ & $6,86 \pm 0,07 \mathrm{ab}$ \\
\hline $\mathrm{T} 10$ & $\mathrm{PC}$ & 85 & 15 & $1,53 \pm 0,12 c$ & $0,46 \pm 0,08$ & & $0,23 \pm 0,06$ abc & $21,0 \pm 1,7 \mathrm{ab}$ & $259,0 \pm 39 \mathrm{~b}$ & $6,93 \pm 0,03 \mathrm{ab}$ \\
\hline $\mathrm{T} 11$ & PC & 80 & 20 & $1,65 \pm 009$ bc & $0,48 \pm 0,06$ & & $0,22 \pm 0,03 \mathrm{abc}$ & $21,0 \pm 5,0 \mathrm{ab}$ & $313,0 \pm 26 \mathrm{ab}$ & $6,94 \pm 0,04 \mathrm{ab}$ \\
\hline $\mathrm{T} 12$ & $\mathrm{PC}$ & 75 & 25 & $1,71 \pm 0,06 \mathrm{~b}$ & $0,52 \pm 0,06$ & & $0,24 \pm 0,02 \mathrm{abc}$ & $21,0 \pm 1,0 \mathrm{ab}$ & $301,7 \pm 42$ a & $6,98 \pm 0,06 \mathrm{a}$ \\
\hline
\end{tabular}




\section{Tabla 3}

Evaluación del color en los tratamientos planteados empleando la tabla Munsell Soil Color Charts (2010) del filtrante elaborado a partir de anís de monte (Tagetes filifolia Lag.) edulcorado con hojas de estevia (Stevia rebaudiana Bertoni)

\begin{tabular}{|c|c|c|c|c|c|}
\hline \multirow{2}{*}{$\begin{array}{l}\text { Trata- } \\
\text { miento }\end{array}$} & \multirow{2}{*}{$\begin{array}{c}\text { Segmento } \\
\text { Planta } \\
\text { Anís de Monte }\end{array}$} & \multicolumn{2}{|c|}{ Formulación (\%) } & \multirow{2}{*}{$\begin{array}{c}\text { Color Tabla } \\
\text { Munsell }\end{array}$} & \multirow[b]{2}{*}{ Color } \\
\hline & & $\mathrm{AM}^{2}$ & $\mathrm{HE}^{2}$ & & \\
\hline T1 & $\mathrm{H}+\mathrm{F}$ & 90 & 10 & $5 \mathrm{Y} 7 / 6 / / 8$ & Amarillo \\
\hline $\mathrm{T} 2$ & $\mathrm{H}+\mathrm{F}$ & 85 & 15 & $5 Y 7 / 8$ & Amarillo \\
\hline T3 & $\mathrm{H}+\mathrm{F}$ & 80 & 20 & $5 Y 7 / 6 / / 8$ & Amarillo \\
\hline $\mathrm{T} 4$ & $\mathrm{H}+\mathrm{F}$ & 75 & 25 & $5 Y 7 / 6 / / 6$ & Amarillo \\
\hline T5 & Tallos & 90 & 10 & $5 Y 7 / 4$ & Amarillo pálido \\
\hline T6 & Tallos & 85 & 15 & $5 Y 7 / 3$ & Amarillo pálido \\
\hline $\mathrm{T} 7$ & Tallos & 80 & 20 & $5 Y 7 / 3$ & Amarillo pálido \\
\hline $\mathrm{T} 8$ & Tallos & 75 & 25 & $5 Y 7 / 3$ & Amarillo pálido \\
\hline T9 & $\mathrm{PC}$ & 90 & 10 & $5 Y 7 / / 4 / 6$ & Amarillo \\
\hline $\mathrm{T} 10$ & $\mathrm{PC}$ & 85 & 15 & $5 Y 8 / 7 / / 6$ & Amarillo \\
\hline T11 & $\mathrm{PC}$ & 80 & 20 & $5 Y 7 / 6 / / 6$ & Amarillo \\
\hline T12 & $\mathrm{PC}$ & 75 & 25 & $5 Y 8 / 7 / / 6$ & Amarillo \\
\hline
\end{tabular}

${ }^{1} \mathrm{H}+\mathrm{F}=$ Hojas más flores $; \mathrm{PC}=$ Planta completa $;{ }^{2} \mathrm{AM}=$ Anís de monte; $\mathrm{HE}=$ Hojas de Estevia.

Con respecto al tiempo de infusión inicial (cuando empiezan a difundir los sólidos solubles del filtrante) y tiempo de infusión final se observa que fue similar en todos los tratamientos evaluados; siendo los tratamientos T2 y T3 los que más se aproximaron a los valores de filtrante de manzanilla comercial Herbi (265 segundos) y Mc Collins (242 segundos); anís comercial Herbi (246 segundos) y Mc Collins (244 segundos). Según la NTC3408 (ICONTEC, 1992) la duración de la infusión de té no debe diferir en seis minutos (360 segundos), en los filtrantes elaborados registraron valores de tiempo de infusión por debajo de los 360 segundos.

El pH de la infusión de filtrante obtenido se observó que al emplear la planta completa en la elaboración del filtrante (Tratamientos T9, T10, T11 y T12) se registró valores de $\mathrm{pH}$ cercanos a $\mathrm{pH}$ neutro, siendo estos tratamientos los que más se aproximaron a los valores de filtrante de manzanilla comercial Herbi $(7,14)$ y Mc Collins $(6,92)$; anís comercial Herbi $(7,13)$ y Mc Collins $(7,09)$ (Tabla2).

En la evaluación sensorial mostrada en la Tabla 4, las mayores calificaciones por parte de los panelistas se registraron en los tratamientos donde se empleó hojas + flores de anís de monte con respecto a planta completa y/o tallo. Con respecto al olor, se puede apreciar que las mayores calificaciones por parte de los panelistas estuvo cuando se empleó hojas + flores, tallos de anís de monte, sobresaliendo el Tratamiento T2 $(5,75)$ y T3 $(5,50)$ con calificaciones Muy Bueno (típico de anís de monte, olores característicos marcados a anís de monte y muy ligero olor a estevia). Vásquez (1987) menciona que empleando una temperatura de $60^{\circ} \mathrm{C}$ en el proceso de secado de hierba luisa era la adecuada para preservar las propiedades aromáticas de la planta, en la presente investigación el empleo de $65^{\circ} \mathrm{C}$ en plantas de anís de monte permitió preservar el aroma, reflejado principalmente en los T2 y T3 donde se registraron las mayores calificaciones del olor del filtrante. El sabor de los filtrantes obtenidos, las mayores calificaciones se registró cuando se empleó hojas + flores de anís de monte, sobresaliendo los Tratamientos T2 $(5,83)$ y T3 $(5,67)$ con calificaciones Muy Bueno (Típico de anís de monte, con un muy ligero sabor a estevia). El dulzor de los filtrantes obtenidos fue apreciado por los panelistas cuando se empleó en proporciones de 15,20 y $25 \%$, siendo mayormente captado cuando se empleó hojas + flores de anís de monte donde se registró los mayores calificaciones, siendo 
los tratamientos T2 $(5,17)$, T3 $(4,58)$ y T4 $(5,00)$ los que obtuvieron calificaciones de Bueno (agradable dulzor, con persistencia a anís de monte y regular persistencia del esteviósido). El aspecto general de los filtrantes obtenidos las mayores calificaciones se obtuvieron cuando se empleó hojas + flores de anís de monte, sobresaliendo los tratamientos T2 $(6,17)$, T1 $(5,83)$ y T4 $(5,58)$ con calificación Muy Bueno (agradable a los ojos, brillo, amarillo oscuro y transparencia adecuada); todos los tratamientos presentaron una ausencia de sólidos en suspensión.

Acuña y Torres (2010), quienes emplearon hojas de estevia en la elaboración de filtrante de jengibre, obtuvieron que al emplear 10 y $20 \%$ de hojas de estevia en el filtrante disminuyó la pungencia del rizoma de jengibre; sin embargo, en el filtrante de anís de monte edulcorado con estevia al emplear 15 y $20 \%$ de hojas de estevia influenció mayormente en el dulzor del filtrante, cuyos panelistas notaron la persistencia del sabor del anís de monte, con una regular persistencia del esteviósido. El tratamiento donde se empleó $80 \%$ de hojas más flores de anís de monte y $20 \%$ de hojas de estevia registró humedad $10,87 \%$, cenizas $9,19 \%$, fibra cruda $10,56 \% \mathrm{BH}$, aceite esencial $0,5 \%$; valores que estuvieron dentro de los rangos establecidos en el NTP 209.244. 1986; a excepción de la humedad que es $9 \%$ como valor máximo.

\section{Conclusiones}

El empleo de 80 a $85 \%$ de hojas + flores de anís de monte y 15 a $20 \%$ de hojas de estevia fueron las formulaciones apropiadas para obtener un filtrante de anís de monte edulcorado con hojas de estevia con un adecuado grado de aceptabilidad.

Los filtrantes elaborados con tallos de anís de monte, mostraron un color amarillo; asimismo, se registró mayores valores de tiempo de infusión (344 segundos) y menores valores en las calificaciones de sabor por parte de los panelistas (5: bueno). La perspectiva futura del producto es emprender una MYPE direccionada al campo de los filtrantes; pues este trabajo es una base para seguir estableciendo formulaciones con nuevas plantas aromáticas nativas con cualidades sensoriales y beneficios funcionales, esto debido a que la región Amazonas es proveedora de un sinnúmero de hierbas con propiedades funcionales aún sin estudiar; además, fomentar la alimentación saludable, disminuyendo el consumo excesivo de azúcares convencionales.

\section{Tabla 4}

Evaluación sensorial de los atributos olor, sabor, dulzor y aspecto general del filtrante elaborado a partir de anís de monte (Tagetes filifolia Lag.) edulcorado con hojas de estevia (Stevia rebaudiana Bertoni)

\begin{tabular}{|c|c|c|c|c|c|c|c|}
\hline \multirow[b]{2}{*}{ Trat } & \multirow{2}{*}{$\begin{array}{c}\text { Segmen- } \\
\text { to } \\
\text { Planta } \\
\text { AM }\end{array}$} & \multicolumn{2}{|c|}{$\begin{array}{l}\text { Formula- } \\
\text { ción }(\%)\end{array}$} & \multicolumn{4}{|c|}{ Evaluación sensorial } \\
\hline & & AM & $\mathrm{HE}$ & Olor & Sabor & Dulzor & $\begin{array}{l}\text { Aspecto } \\
\text { General }\end{array}$ \\
\hline $\mathrm{T} 1$ & $\mathrm{H}+\mathrm{F}$ & 90 & 10 & $5,17 \pm 1,5$ abc & $5,50 \pm 1,4$ & $3,83 \pm 2,0$ abcd & $5,83 \pm 1,2 \quad a$ \\
\hline $\mathrm{T} 2$ & $\mathrm{H}+\mathrm{F}$ & 85 & 15 & $5,75 \pm 1,1 \quad$ a & $5,83 \pm 0,9$ & $5,17 \pm 1,3$ a & $6,17 \pm 0,8$ \\
\hline $\mathrm{T} 3$ & $\mathrm{H}+\mathrm{F}$ & 80 & 20 & $5,50 \pm 1,4 \quad a b$ & $5,67 \pm 1,2$ & $4,58 \pm 1,5 \mathrm{ab}$ & $5,42 \pm 1,2$ \\
\hline $\mathrm{T} 4$ & $\mathrm{H}+\mathrm{F}$ & 75 & 25 & $5,17 \pm 1,6 \quad a b c$ & $5,25 \pm 1,4$ & $5,00 \pm 1,5$ a & $5,58 \pm 0,9$ \\
\hline T5 & Tallos & 90 & 10 & $4,33 \pm 1,4 \quad a b c$ & $4,58 \pm 0,8$ & $3,25 \pm 1,6$ bcd & $5,17 \pm 1,1$ \\
\hline T6 & Tallos & 85 & 15 & $4,33 \pm 1,9 \quad a b c$ & $4,67 \pm 1,0$ & $3,83 \pm 1,9$ abcd & $5,58 \pm 0,8$ \\
\hline $\mathrm{T} 7$ & Tallos & 80 & 20 & $4,50 \pm 1,5 \quad a b c$ & $4,92 \pm 1,4$ & $4,50 \pm 1,8 \mathrm{ab}$ & $4,42 \pm 1,7$ \\
\hline $\mathrm{T} 8$ & Tallos & 75 & 25 & $4,42 \pm 0,8$ & $5,42 \pm 0,9$ & $4,33 \pm 1,8$ abc & $5,25 \pm 1,4$ \\
\hline T9 & PC & 90 & 10 & $4,42 \pm 1,8$ & $4,58 \pm 1,4$ & $2,50 \pm 1,6 \mathrm{~d}$ & $5,17 \pm 1,5$ \\
\hline $\mathrm{T} 10$ & $\mathrm{PC}$ & 85 & 15 & $3,67 \pm 1,3$ & $4,33 \pm 0,7$ & $2,83 \pm 1,3 \mathrm{~cd}$ & $5,42 \pm 1,0$ \\
\hline $\mathrm{T} 11$ & $\mathrm{PC}$ & 80 & 20 & $4,08 \pm 1,1 \quad b c$ & $4,58 \pm 0,9$ & $2,75 \pm 1,1 \mathrm{~cd}$ & $5,42 \pm 0,7$ \\
\hline $\mathrm{T} 12$ & $\mathrm{PC}$ & 75 & 25 & $5,17 \pm 0,9 \quad a b c$ & $5,33 \pm 1,2$ & $4,50 \pm 1,8 \mathrm{ab}$ & $5,08 \pm 1,4 \quad a b$ \\
\hline
\end{tabular}

${ }^{1} \mathrm{H}+\mathrm{F}=$ Hojas más flores $; \mathrm{PC}=$ Planta completa $;{ }^{2} \mathrm{AM}=$ Anís de monte HE = Hojas de Estevia. 


\section{Referencias bibliográficas}

Acuña, O.; Torres, A. 2010. Aprovechamiento de las propiedades funcionales del jengibre (Zingiber officinale) en la elaboración de condimento en polvo, infusión filtrante y aromatizante para quema directa. Revista Politécnica 29: 60-69.

AOAC International. 2005. Official methods of analysis. 18th ed. Official method 920.100A fiber (crude) in plants. Gaithersburg Md, USA.

AOAC International. 2012. Official methods of analysis. 19th ed. Official method 930.10 ash content. Gaithersburg Md, USA.

Barba, F.J.; Criado, M.N.; Belda-Galbis C.M.; Esteve, M.J.; Rodrigo, D. 2014. Stevia rebaudiana Bertoni as a natural antioxidant/antimicrobial for high pressure processed fruit extract: Processing parameter optimization. Food Chemistry 48: 261-267.

Belda-Galbis, C.M.; Pina-Pérez, M.C.; Espinosa, J.; Marco-Celdrán, A. 2014. Use of the modified Gompertz equation to assess the Stevia rebaudiana Bertoni antilisterial kinetics. Food Microbiology 38: 56-61.

Campos, M.; Medina, J.L.; Serrato, M.A. 2010. Aspectos agronómicos para la producción de anís de monte (Tagetes filifolia Lag.) en Temporal en Ociutuco, Morelos. Rev. Fitotec. Mex. 33: 97-105.

Carbonell-Capella, J.M.; Barba, F.J.; Esteve, M.J.; Frígola, A. 2013. High-pressure processing of fruit juice mixture sweetened with Stevia rebaudiana Bertoni: optimal retention of physical and nutritional quality. Innovate Food Science \& Emerging Technologies 18: 48-56.

Cerruti, R.R.; Wang, K.; Ploeg, A.; McSorley, R. 2010. Using marigold (Tagetes spp.) as a cover crop to protect crops from plant-parasitic nematodes. Applied Soil Ecology 46: 307-320.

Follegatti, L.M. 2002. Formulación y evaluación sensorial de mezclas de manzanilla común (Matricaria chamomilla L.) y hierba luisa (Cymbopogon citratus (DC.) Stapf.) conteniendo corteza de uña de gato (Uncaria tomentosa (Wild.) DC.) para uso en infusiones. Tesis Maestría Tecnología de Alimentos, UNALM, Lima - Perú.
ICONTEC. 1992. Norma Técnica Colombiana NTC3408. Plantas aromáticas. Té negro. Preparación de la infusión para uso en análisis sensorial. Instituto Colombiano de Normas Técnicas y Certificación (ICONTEC), Bogotá, Colombia.

Lemus-Moncada, R.; Vega-Gálvez, A.; Zura-Bravo, L.; Kong, A.H. 2012. Stevia rebaudiana Bertoni, source of a high-potency natural sweetener: A comprehensive review on the biochemical, nutritional and functional aspects. Food Chemistry 132: 1121-1132.

Munsell® Color Company-x-Rite. 2010. Munsell SoilColor Chart (2009 Year Revised - 2010 Production).

Nolazco, D.M. 2008. Obtención de un filtrante de maíz morado (Zea mays L.) evaluación de pérdida de color y degradación de antocianinas en el almacenaje. Tesis Maestría Tecnología de Alimentos, UNALM, Lima, Perú.

NTP 209.244: 1986. Hierba luisa en bolsas filtrantes. INDECOPI. (Revisada 2010)

Olsen, E.D. 1986. Métodos ópticos de análisis. Edit. Reverté S.A.

Ortíz, M.; Yon, M.J.; Ortíz, Z. 2006. Industrialización de la hoja de Sambucus peruviana (sauco), preparación de formas medicamentosas: bolsitas filtrantes y cápsulas. Sciendo 9: 1-5.

Serrato-Cruz; M.A.; Díaz-Cedillo, F.; Barajas-Pérez, J.S. 2008. Composición del aceite esencial en germoplasma de Tagetes filifolia Lag. de la región Centro-Sur de México. Agrociencia 42: 277-285.

Vásquez, E.R.; Millones, C.E.; Tafur, A.R.; Salazar, S.M.; Mori, G.H.; Bacalla, J. 2011. Actividad antibacteriana del aceite esencial de anís de la sierra (Tagetes filifolia Lag.) sobre algunas bacterias de interés alimentario. Ciencia, Tecnología y Humanidades 2: 31-35.

Vásquez, J.G. 1987. Procesamiento de hierba luisa (Cymbopogon citratus) en bolsas filtrantes. Tesis Ing. Industrias Alimentarias, UNALM, Lima - Perú. 